\title{
Aplicación del Ultrasonido en la Industria de los Alimentos
}

\section{Application of Ultrasound in the Food Industry}

\author{
Delgado Javier Orlando \\ Escuela Ciencias Básicas Tecnología e Ingeniería, Universidad Nacional Abierta y a Distancia-UNAD, \\ Bogotá, Colombia, jodelgado@unadvirtual.edu.co
}

Recibido: 17/09/2011 - Aprobado:15/12/2011

\section{RESUMEN}

El ultrasonido es una de las tecnologías emergentes con más investigación y desarrollo para la conservación de alimentos, utilizada, principlamente para la disminución de la concentracion de microorganismos y la inhibicion de la actividad enzimática, sin alterar las propiedades físicas, químicas y nutricionales de los alimentos.

Gracias al análisis de direfentes fuentes bibliográficas, se logró elaborar este documento en el que se destacan las aplicaciones del ultrasonido en los principales procesos de la tecnología de alimentos, incluyendo los beneficios del efecto de la cavitación, la intensidad y las frecuencias aplicadas, en cada una de las investigaciones que se han realizado actualmente.

Palabras clave: cavitación, conservación, frecuencia, intensidad, propiedades, tecnología, ultrasonido

\section{Abstract}

Ultrasound is an emerging technology with more research and development for food preservation, one of the qualities of is the reduction of the concentration of microorganisms, inhibition of enzyme activity without altering the physical, chemical and nutritional foods.

It was conducted direffent literature sources analysis to develop a document with ultrasound applications in main food technology processing, the benefits of cavitation effect, intensity and frequency applied in each of researching works that have been made today.

Keywords: cavitation intensity, conservation frequency, intensity, property, technology, ultrasound 


\section{InTRODUCCIÓN}

Ultrasonido es el nombre que reciben las ondas de sonido con frecuencias mayores a aquellas que pueden ser detectadas por el oído humano. El ultrasonido maneja ondas con frecuencias entre $16 \mathrm{KHz}$ y $20 \mathrm{MHz}$, las cuales al ser aplicadas pueden llegar hasta $5 \mathrm{MHz}$ en gases o hasta $500 \mathrm{MHz}$ en líquidos y sólidos[1], [2], [3].

El ultrasonido es una tecnología emergente que, recientemente, ha sido estudiada para propósitos de inactivación microbiana y enzimática, pero que durante años ha sido objeto de investigación en la industria de alimentos, especialmente, en el tema de control de calidad. Se ha demostrado que puede ser utilizado para la evaluación de textura, composición y viscosidad de alimentos[4], para el desarrollo de técnicas de análisis no invasivas y para determinar el nivel de homogenización de glóbulos de grasa en leche[5], entre otras aplicaciones. Adicionalmente, el ultrasonido, por su capacidad para destruir paredes y membranas biológicas, se considera una tecnología promisoria tanto para la destrucción de microorganismos a temperaturas de procesamiento inferiores a las utilizadas durante la esterilización, como para acompañar otras tecnologías de proceso como lo son la extracción, las altas presiones, la pasteurización, entre otras [6], [7], [8], [9], [10].

Dependiendo de los rangos de frecuencias utilizados, el ultrasonido se puede dividir en dos tipos: ultrasonido de baja intensidad y ultrasonido de alta intensidad, tabla 1.

TABLA I

Clasificación del Ultrasonido

\begin{tabular}{l|l|l}
\hline ULTRASONIDO & $\begin{array}{l}\text { ALTA INTENSIDAD } \\
\text { 18-100kHz. } \\
\text { Procesamiento o estabilización } \\
\text { de alimentos }\end{array}$ & $\begin{array}{l}\text { Rompimiento celular } \\
\text { Permeabilización la de membrana } \\
\text { celular } \\
\text { Cambios estructurales y } \\
\text { Fisicoquímicos } \\
\text { Homogenización de emulsiones }\end{array}$ \\
$\begin{array}{l}\text { BAJA INTENSIDAD } \\
\text { >100kHz } \\
\text { Diagnostico, control de calidad, } \\
\text { fenomenos de relajación }\end{array}$ & $\begin{array}{l}\text { Ecografías } \\
\text { Aplicaciones terapéuticas }\end{array}$ \\
\hline
\end{tabular}

Fuente: autor

\section{A. Mecanismos de acción del ultrasonido}

Las investigaciones actuales se han concentrado en entender el mecanismo de acción del ultrasonido de alta intensidad para la inactivación de microorganismos, ya que cuando este se aplica, tiene el potencial de causar la muerte celular, lo que conlleva a diversas hipótesis, entre las que se encuentran los fenómenos de cavitación, el calentamiento localizado, la formación de radi- cales libres y el aumento de la temperatura y la presión, entre otras [11], [12], [13].

Los mecanismos de acción del ultrasonido se clasifican principalmente en térmicos (generación de energía calórica o mecánica), y no térmicos (cavitación, rarefacción, formación de radicales libres, choques micro-mecánicos y fuerza de radiación), como se muestra en la tabla 2. 
TABLA ॥

Mecanismos de Acción del Ultrasonido

\begin{tabular}{|c|c|c|}
\hline TEORÍA & MECANISMO & APLICACIÓN \\
\hline Cavitación & $\begin{array}{l}\text { Remoción mecánica por irrupción o atrapamiento } \\
\text { de la bacteria, a través de burbujas localizadas } \\
\text { con altas temperaturas }\left(5500^{\circ} \mathrm{C}\right) \text { y presiones } \\
(500 \mathrm{MPa}) \text {. }\end{array}$ & $\begin{array}{l}\text { Procesos de desinfección, } \\
\text { lisis celular e inactivación } \\
\text { enzimática. }\end{array}$ \\
\hline $\begin{array}{l}\text { Formación } \\
\text { de Radicales } \\
\text { Libres }\end{array}$ & $\begin{array}{l}\text { Sonólisis del agua puede producir iones }(\mathrm{OH}-) \\
\text { y }(\mathrm{H}+) \text { y peróxido de hidrogeno. }\end{array}$ & $\begin{array}{l}\text { Inactivación microbiana y } \\
\text { enzimática. }\end{array}$ \\
\hline $\begin{array}{l}\text { Choques } \\
\text { micro-mecánicos } \\
\text { intracelulares }\end{array}$ & $\begin{array}{l}\text { Disrupción de las células y disminución del grosor } \\
\text { de paredes celulares. }\end{array}$ & $\begin{array}{l}\text { Lisis celular, inactivación } \\
\text { enzimática y efecto } \\
\text { antimicrobiano. }\end{array}$ \\
\hline $\begin{array}{l}\text { Generación de } \\
\text { energía calórica } \\
\text { y mecánica }\end{array}$ & $\begin{array}{l}\text { Propagación de la onda; decrece con la distancia } \\
\text { al ser aplicado en material atenuante. Esta porción } \\
\text { que es absorbida se traduce en calor. }\end{array}$ & Inactivación microbiana. \\
\hline Fuerza de radiación & $\begin{array}{l}\text { Movimiento o vibración local de un tejido por } \\
\text { fuerza de radiación acústica. }\end{array}$ & Análisis clínicos. \\
\hline $\begin{array}{l}\text { Compresión } \\
\text { y Rarefacción }\end{array}$ & Microcorriente acústica. & $\begin{array}{l}\text { Inactivación microbiana } \\
\text { y enzimática. }\end{array}$ \\
\hline
\end{tabular}

\section{B. Ventajas y desventajas de la técnica del ultrasonido.}

\section{1) En la salud humana}

- El ultrasonido, por ser una técnica no destructiva de tejidos, no produce ninguna reacción secundaria comprobada, al ser humano; los alimentos tratados no producen modificaciones genéticas

- Mejora la digestibilidad de proteínas gracias al proceso de cavitación y al rompimiento de cadenas de aminoácidos no digeribles por algunas personas. Las propiedades nutricionales de los alimentos se mantienen estables, y su margen de calidad, alta.

- No se debe confundir los tratamientos de ultrasonidos de potencia con ultrasonidos de señal. Los ultrasonidos de potencia utilizados en alimentos tienen rangos de frecuencia entre $20 \mathrm{kHz}$ y $100 \mathrm{KHz}$; si se somete a alimentos, con frecuencias más altas, se estaría tratando entonces con frecuencias de señal que sí pueden producir radicales libres en aceites y la destrucción de células tanto benéficas como no benéficas para el organismo.

\section{2) Procesamiento de alimentos}

Como desventaja se tiene que el efecto del ultrasonido en grandes cantidades de prducción no es totalmente eficiente y es necesario la combinación con tratamientos de calor, presión o el uso de los dos.

- No se ha profundizado en factores clave de diseño y su posterior escalado.

- Su efecto en esporas es muy efectivo, pero se requiere de mucho de tiempo.

- La técnica es óptima para mejorar cualidades como emulsificación, viscosidad, desaireado en líquidos, inactivación de enzimas y vida útil. 


\section{Aplicaciones del ultrasonido}

1) Ultrasonido como método de emulsificación/homogenización. Cuando dos líquidos inmiscibles se irradian con ultrasonidos, no siempre es posible mezclarlos y crear una emulsión, ya que una de las fases debe cavitar y de este modo poder hacer miscibles las dos fases.

Durante la cavitación, las burbujas generadas colapsan cerca a la interface existente entre los dos líquidos y este choque resulta en la mezcla eficiente de las dos fases [14].

2) Filtración asistida ultrasónicamente: la aplicación de energía ultrasónica en la superficie de la membrana utilizada, puede aumentar el flujo del líquido, ya que la permeabilidad intrínseca de esta, se ve afectada por las ondas de ultrasonido [15], [16]. La filtración asistida ultrasónicamente se ha aplicado con éxito para aumentar la filtración de aguas industriales residuales, consideradas difíciles de procesar[17], [18], [15]. Sin embargo, en este proceso, se debe controlar la intensidad del ultrasonido, con el fin de prevenir el daño de la membrana de filtración [4].

\section{3) Ultrasonido como método antiespuman-} te: ondas de ultrasonido de alta intensidad ofrecen un método atractivo para controlar el exceso de espuma en los procesos, y al mismo tiempo brindan condiciones estériles, lo que hace que este método sea particularmente apropiado para su implementación en las industrias alimentaria y farmacéutica [19]. Como ejemplo, se encuentra el control del exceso de espuma en el embotellado de alta velocidad en líneas de latas de bebidas carbónicas, ya que debido a la cavitación (ruptura de burbujas) se controla, casi instantáneamente, bajo el haz acústico formado por estas ondas de ultrasonido [19].

4) Ultrasonido en el proceso de desgasificación/de-aireación: en el procesamiento de las bebidas carbonatadas, el objetivo es desplazar el aire de la superficie del líquido, con el fin de evitar daños organolépticos del producto por las bacterias y el oxígeno. Este proceso consiste en el acoplamiento de un transductor en la parte exterior de la botella, lo que lleva a la desgasificación.

5) Despolimerización por ultrasonido: la aplicación de ultrasonido de baja potencia, por lo general, causa una reducción temporal de la viscosidad de los líquidos poliméricos, mientras que tratamientos con altas potencias generan despolimerización y cambios permanentes en la reología de un producto. Estas modificaciones, atribuidas al tratamiento ultrasónico, permiten generar productos con características fisicoquímicas y organolépticas diferentes, motivo por el cual esta tecnología se utiliza en el procesamiento de bebidas y en el desarrollo de nuevos productos [20], [21].

6) Ultrasonido y cocción: se ha comprobado que la aplicación de ultrasonido en el proceso de cocción genera una mayor retención de la humedad de los productos tratados, así como una mayor eficiencia energética, lo que optimiza los tratamientos convencionales solo con temperatura. Esta combinación de tecnologías podría considerarse como una técnica nueva, rápida y energéticamente eficiente, que puede mejorar los atributos de textura de productos como la carne cocida [22].

7) Ultrasonido y corte: el corte de productos alimenticios mediante la aplicación de ondas de vibración ultrasónica, se ha convertido en un procedimiento de gran impacto en la industria, el cual compite directamente con las tecnologías tradicionales como son el corte de alta velocidad y el uso de sierras y cuchillos [23]. Se ha comprobado que mediante el proceso ultrasónico se obtiene mayor precisión en los cortes y se produce un menor porcentaje de desperdicios. La aplicación más difundida se encuentra en el corte de alimentos frágiles como tartas, pasteles y otros productos de panadería, así como en productos grasos (quesos) o viscosos como las gelatinas [24]. 
8) Ultrasonido para mejorar viscosidad y textura: en la industria alimentaria, el ultrasonido se utiliza cada vez más para la emulsificación de productos como jugos de frutas, mayonesas y salsas de tomate[14]; para la homogeneización de la leche [25] y para la encapsulación de aromas[26], así como para la emulsión de aceites comestibles [27]. Esta predilección por el método ultrasónico se debe a que requiere menos energía para producir una emulsión, por lo cual es más económico que los métodos convencionales.

\section{9) Ultrasonido en el proceso de congelación} y cristalización: bajo la influencia del ultrasonido, la congelación proporciona un tamaño final de los cristales de hielo, menor, y el daño celular se reduce significativamente [28] ya que el enfriamiento acelerado se logra mediante la mejora de la transferencia de calor, lo cual protege, en mayor proporción, la estructura del alimento tratado [29].

10) Ultrasonido en el proceso de descongelación: para la descongelación de carnes y pescados, los métodos ultrasónicos son más eficientes que los métodos comunes, ya que se logra disminuir el tiempo de esta, lo que, a su vez, reduce las pérdidas por goteo y genera una mejora de la calidad del producto final [30].

11) Ultrasonido en el proceso de secado: el secado acústicamente asistido ha sido un tema de interés por muchos años [31]. Los métodos tradicionales para la deshidratación de alimentos por una corriente forzada de aire caliente son bastante económicos, pero la eliminación de la humedad interior toma un tiempo relativamente largo.

La tecnología ultrasónica de deshidratación osmótica utiliza temperaturas más bajas, y evita una mayor pérdida de agua y las tasas de ganancia de soluto [32]. Debido a las bajas temperaturas durante la deshidratación y los tiempos de tratamiento más cortos, cualidades de los alimentos, tales como sabor, color y valor nutritivo, se mantienen inalteradas.
12) Inactivación microbiana y de enzimas. La pasteurización térmica convencional y la esterilización son las técnicas más comunes en la actualidad para inactivar los microorganismos y las enzimas en productos alimenticios. Sin embargo, las intensidades de temperatura de tratamiento y el tiempo del proceso también son proporcionales a la cantidad de pérdida de nutrientes, el desarrollo de sabores indeseables y el deterioro de las propiedades funcionales de los alimentos. De esta manera, el ultrasonido proporciona un método para mejorar dichos procesos en virtud de los efectos de la cavitación [33].

\section{Equipos de ultrasonido}

En la actualidad, existen tres tipos de equipos diseñados para la aplicación de tratamientos por ultrasonido en el laboratorio: el baño de ultrasonido, el "cup-horn", sonicador y el de inmersión directa de la sonda de ultrasonido [34].

\section{1) Baños de ultrasonidos}

Los baños de ultrasonidos posiblemente sean la aplicación más popular de los ultrasonidos de potencia. Aunque se utilicen normalmente como equipos de limpieza de material de laboratorio, en un gran número de referencias bibliográficas se encuentra que estos se han usado para acelerar los procesos de transferencia de materia en medios líquidos [35]. Se pueden calificar como equipos ultrasónicos simples y compactos; los transductores colocados en la base de un recipiente de acero inoxidable y con el sistema de generación y sus principales limitaciones como es la poca potencia que suministran al medio si se comparan con otros sistemas, como la variación del campo acústico dentro del baño y la dificultad en el control de la temperatura [36].

\section{2) Sistemas tipo sonda}

En este tipo de equipos, se utiliza una sonda metálica para aplicar al medio líquido la vibración generada en el transductor. La potencia aplicada dependerá de la amplitud de vibración en la punta de la sonda que se controlará variando la potencia eléctrica suministrada por el equipo generador/amplificador. 
Tanto el diseño como la forma de la sonda tienen mucha importancia. La longitud de la misma debe de ser de media longitud de onda o múltiplo de dicha longitud. Las sondas cilíndricas se limitan a transmitir la energía acústica al medio por tratar. Sin embargo, si a lo largo de la sonda hay reducción del diámetro, entonces se produce una ganancia en la amplitud de vibración [37]. Muchos sistemas se diseñan para operar con sondas intercambiables de diferentes diámetros de superficie emisora [38].

El material utilizado para la fabricación de las sondas debe tener una alta resistencia a la fatiga y a la erosión que producen la cavitación en la punta de la sonda y las bajas pérdidas acústicas [39].

\section{3) Equipos diseñados para la aplicación de ultrasonido de potencia.}

En la actualidad, se han diseñado y modificado equipos, con el fin de realizar el efecto del ultrasonido en diferentes alimentos. Es así, como secadores de convección directa con transductores ultrasónicos se han utilizado para el secado de algunas frutas y verduras Fig. 1. El secador consta de un plato vibrante donde se ubican las láminas del alimento; el aire caliente se empuja para realizar el secado y el transductor produce vibraciones a la estructura, mejorando la extracción de agua en el producto [40].

Otra propuesta de diseño es ubicar transductores en un túnel con doble camisa A, B y C; D sería un cilindro giratorio por donde pasa el fluido y $\mathrm{E}$, el líquido receptor de la onda ultrasónica, los cuales se utilizarían para la refrigeración y pasteurización de la concentracion de zumos y pulpas de frutas [17].
Los dispositivos tipo túnel se combinan con métodos térmicos ya que no se han diseñado equipos para tratar volúmenes industriales; a los equipos de pasteurización se le han acoplado sondas para aplicar a la leche el tratamiento o se ubican los equipos tipo túnel al final del pasteurizador. Estos pasteurizadores tienen como ventaja que la temperatura del tratamiento térmico disminuye y se complementa con una onda ultrasónica de $20 \mathrm{KHz}$ de potencia, intensidad adecuada para el rompimiento celular de microorganismos patógenos.

En zumos de frutas, la pasteurización es similar; variables como el pH y la acidez se ven afectadas por el proceso. Para evitar modificaciones en las mismas, se recomienda disminuir el tiempo y la temperatura del tratamiento, porque reduce la calidad del producto. Entonces, la ventaja de aplicar una radiación ultrasónica para reducir la microbiota, radica en que el $\mathrm{pH}$ y la acidez no varían y, por el contrario, se mejora la viscosidad en productos concentrados como la mermelada.

\section{Resultados}

\section{A. Aplicación del ultrasonido de potencia en la industria de leches y derivados}

El efecto del ultrasonido en el tratamiento de leches y derivados ha sido combinado con tecnologías actuales térmicas de procesamiento; lo que se busca en estas materias primas es la reducción de la microbiota que altera las características de procesamiento y atenta contra la salud humana. El ultrasonido, por ser una tecnología no destructiva, puede mejorar los procesos, las características nutricionales, la digestibilidad al actuar sobre cadenas largas de aminoácidos y los procesos de homogenización, como se muestra en la tabla 3. 
TABLA III

Efecto del Ultrasonido en los Procesos de Leches y Derivados

\begin{tabular}{|c|c|c|}
\hline PRODUCTO & OPERACIÓN/PROCESO & EFECTO DEL ULTRASONIDO \\
\hline Leche & $\begin{array}{l}\text { Pasteurización } \\
\text { y homogenización }\end{array}$ & $\begin{array}{l}\text { Inactivación de enzimas como la coagulasa positiva. } \\
\text { Rompimiento de los glóbulos grasos aumentado la } \\
\text { homogenización. } \\
\text { Aumenta la digestibilidad de la grasa láctea. }\end{array}$ \\
\hline \multirow{2}{*}{ Quesos } & & $\begin{array}{l}\text { A frecuencias de } 20 \mathrm{kHz} \text { aumenta la penetración } \\
\text { de la salmuera. }\end{array}$ \\
\hline & Emulsificación & $\begin{array}{l}\text { En procesos de atrapamiento de la grasa por el } \\
\text { caseinato se obtiene mejora en la emulsificación. }\end{array}$ \\
\hline Sorbetes & Homogenización & $\begin{array}{l}\text { Intensidades entre } 15 \mathrm{kHz} \text { mejoran las características } \\
\text { de viscosidad. }\end{array}$ \\
\hline Productos evaporados & Antiespumante & $\begin{array}{l}\text { El rendimiento de leches evaporadas y } \\
\text { condensadas dulces. }\end{array}$ \\
\hline Yogur y kumis & Pasteurización & Mejora las características de viscosidad. \\
\hline
\end{tabular}

Fuente el autor

A escala industrial, la leche es la que más ha sido investigada por el efecto del ultrasonido en su composición; se han desarrollado equipos de flujo continuo por ultrasonido en los que se han alcanzado temperaturas de $70^{\circ} \mathrm{C}$. Dicho sistema permite llevar a cabo inactivaciones eficaces de microorganismos tales como Pseudomonas fluorescens y Streptococcus thermophilus en sistemas modelos. [19].
B. Aplicación del ultrasonido de potencia en la industria de frutas, verduras, tubérculos y derivados

Este grupo de alimentos, por su gran contenido de agua, es susceptible a cambios por fermentación, pardeamiento enzimático, bajos rendimientos en emulsificación y secado; el ultrasonido ha sido aplicado a frutas, verduras y a algunos tubérculos, teniendo resultados para la industrialización del ultrasonido en este campo ver tabla 4.

TABLA IV

Efecto del Ultrasonido en Frutas, Verduras y Tubérculos

\begin{tabular}{|c|c|c|}
\hline PRODUCTO & OPERACIÓN/PROCESO & EFECTO DEL ULTRASONIDO \\
\hline Papa & $\begin{array}{l}\text { Pos cosecha } \\
\text { Extracción }\end{array}$ & $\begin{array}{l}\text { Inactiva las enzimas polifenoloxidasa evitando } \\
\text { el pardeamiento enzimático. } \\
\text { Aumenta la extracción de almidón de papa en un 30\% }\end{array}$ \\
\hline Mora, fresa y tomate & Inactivación enzimática & Inactivación en un 90\% de las enzimas. \\
\hline
\end{tabular}

Fuente El autor 


\section{C) Aplicación del ultrasonido de potencia en la industria de carnes y derivados.}

Como se muestra en la tabla 5, los procesos en la tecnología cárnica se centran en el mejoramiento de la maduración de la carne y en la estabilización de emulsiones cárnicas, por medio del aumento de rendimientos en los productos tipo emulsión. Los transductores de ultrasonido hacen que las pequeñas gotas de grasa se puedan mezclar mejor con las proteínas, ya que su efecto dispersa las gotas y emulsifica las proteínas, haciendo que su parte hidrófila sea mayor para el proceso de emulsificación. Otro proceso interesante es la termosonicación, el cual aumenta la vida útil de los productos cárnicos, no excediendo las temperaturas a más de $65^{\circ} \mathrm{C}$, temperatura en la que se descomponen las proteínas cárnicas.

TABLA $\mathrm{V}$

Efecto del Ultrasonido en Carnes

y Derivados Cárnicos.

\begin{tabular}{|l|l|l}
\hline PRODUCTO & $\begin{array}{c}\text { OPERACIÓN/ } \\
\text { PROCESO }\end{array}$ & $\begin{array}{c}\text { EFECTO DEL } \\
\text { ULTRASONIDO }\end{array}$ \\
\hline $\begin{array}{l}\text { Productos } \\
\text { emulsificados } \\
\text { (salchichas) }\end{array}$ & Emulsificación & $\begin{array}{l}\text { Mejora la emul- } \\
\text { sificación entre } \\
\text { proteina y grasa }\end{array}$ \\
\hline $\begin{array}{l}\text { Carnes en } \\
\text { canal de } \\
\text { bovino y } \\
\text { bufalo }\end{array}$ & $\begin{array}{l}\text { Maduración } \\
\text { Emulsificación }\end{array}$ & $\begin{array}{l}\text { Dispersa las } \\
\text { emulsiones }\end{array}$ \\
\cline { 2 - 3 } & Corte & $\begin{array}{l}\text { Mejora la calidad } \\
\text { del corte, }\end{array}$ \\
\hline
\end{tabular}

Fuente El autor

\section{Aplicación del ultrasonido de potencia en la industria de cereales, aceites y derivados.}

En los procesos de la tecnología de cereales y aceites, estos se ven beneficiados en la fermentación, aumento de rendimiento de los aceites y extracción de almidones como se muestra en la tabla 6.
TABLA VI

Efecto del Ultrasonido en Cereales y Derivados

\begin{tabular}{l|l|l} 
PRODUCTO & $\begin{array}{c}\text { OPERACIÓN/ } \\
\text { PROCESO }\end{array}$ & $\begin{array}{l}\text { EFECTO DEL } \\
\text { ULTRASONIDO }\end{array}$ \\
\hline Maíz, trigo & $\begin{array}{l}\text { Extracción } \\
\text { Rompimiento del } \\
\text { almidón; ,mejora } \\
\text { el rendimiento de } \\
\text { en un 85\% }\end{array}$ \\
\hline Aceites & $\begin{array}{l}\text { Reacciones } \\
\text { químicas: } \\
\text { hidrogenación } \\
\text { transesterificación }\end{array}$ & $\begin{array}{l}\text { Mejoramiento de } \\
\text { las reacciones } \\
\text { para la } \\
\text { producción de } \\
\text { biodiesel }\end{array}$ \\
Panes & Saponificación & $\begin{array}{l}\text { Aumenta la } \\
\text { emulsificación d }\end{array}$ \\
\hline Fermentación & $\begin{array}{l}\text { Desgasificación } \\
\text { aumentando el } \\
\text { volumen del pan }\end{array}$ \\
\hline
\end{tabular}

Fuente El autor

\section{E. Aplicación del ultrasonido de potencia en procesos fermentativos y desgasificación de líquidos}

Los ultrasonidos también pueden afectar las reacciones biológicas. Por ejemplo, para la producción de leche fermentada, se desarrolló el método con lactosa hidrolizada, a través de la aplicación de ultrasonidos [41].

El método airbone, entonces, consiste en aplicar ondas ultrasónicas por medio de aire con el propósito de eliminar la espuma en bebidas carbonatadas, procesos de fermentación y otros en que las espumas afectan la calidad del producto. Sin embargo, por problemas de rendimiento, este sistema es poco utilizado. Algunas investigaciones realizadas afirman que aunque la onda ultrasónica no se propaga en forma eficiente en el aire, su efecto de cavitación es lo suficientemente grande para realizar el proceso antiespumante [42]. 
[43] mostraron un aumento en la velocidad de fermentación de sake, cerveza y vino cuando se aplicó ultrasonido de baja intensidad; el efecto fue de desgasificación del $\mathrm{CO} 2$, el cual, normalmente, inhibe el proceso.

\section{F. Aplicación del ultrasonido en transferen- cia de calor}

Los ultrasonidos de potencia se han utilizado para mejorar mecanismos de transferencia de calor tanto por conducción [39] como por convección [22]. El efecto de los ultrasonidos se ha mostrado más intenso en medios líquidos que en gases [44]. Dentro de este campo, resultan especialmente interesantes las aplicaciones en procesos de congelación y descongelación de alimentos [34] y la aplicación de ultrasonidos en la congelación de patatas por inmersión [45]. En los resultados de esta investigación se evidencia un cambio de fase más rápido en presencia de un campo acústico, que fue aumentando hasta un determinado umbral de potencia ultrasónica aplicada, a partir del cual, el incremento de temperatura, provocado por los ultrasonidos, fue significativo y dificultó la congelación. La estructura celular de las muestras tratadas con ultrasonidos resultó mejor, probablemente, debido al rápido enfriamiento [46]. También puede deberse a la presencia de cristales de hielo de menor tamaño como consecuencia del mayor número de puntos de nucleación, debido a la presencia de burbujas de cavitación y a la rotura de los cristales más grandes por el efecto de la vibración [32].

\section{G. Aplicación del ultrasonido en la inactiva- ción de microorganismos y enzimas}

El ultrasonido de alta intensidad se ha utilizado para reducir la carga microbiana en varios tipos de alimentos, debido a la alteración de la membrana celular de los microorganismos, lo cual afecta los procesos homeostáticos y, por lo tanto, su integridad. El uso del ultrasonido para el rompimiento de células es un método de laboratorio estandarizado, cuya eficiencia de lisis es de casi el $100 \%$. El efecto bactericida del ultrasonido se atribuye, generalmente, a la cavitación intracelular. Estos shocks micro-mecánicos alteran componentes estructurales y funcionales de la célula hasta el punto de generar lisis celular. Sin embargo, este tratamiento de sonicación se lleva a cabo para volúmenes pequeños y en condiciones controladas de temperatura. Para condiciones industriales de procesamiento de alimentos aparecen factores que previenen se alcance tal eficiencia.[47], [48].

Mediante el tratamiento por ultrasonido durante un tiempo de 10 minutos, una reducción de aproximadamente 4,0 ciclos y 0,8 ciclos logarítmicos para Salmonella en agua de peptona y leche achocolatada, respectivamente [48], reportó la reducción de la concentración en Salmonella en piel de pollo sumergida en agua clorinada en un rango de 2,5 a 4,0 ciclos logarítmicos. También se ha estudiado el uso del ultrasonido asistido por temperaturas inferiores a $62^{\circ} \mathrm{C}$, logrando una reducción del valor para S. aureus del $63 \%$ en buffer de fosfato y del $43 \%$ en leche UHT [21]. Los tratamientos de ultrasonido durante 300 segundos a potencias de $24,6 \mathrm{~W}$ y $42,0 \mathrm{~W}$ reducen la concentración de E. coli en 1.0 y 2.0 ciclos logarítmicos, respectivamente [48].

\section{H. Tiempo de acción e intensidades utilizadas en la aplicación de ultrasonido en alimentos.}

Para aplicar el ultrasonido y todos sus beneficios, es de gran importancia saber las intensidades, el tiempo y el equipo que se debe utilizar para cada tipo de alimento. En la tabla 7 se muestran las condiciones favorables de algunos de los alimentos investigados, los cuales han tenido resultados favorables, sin modificar sus propiedades físicas y químicas. 
TABLA VII

CONDICIONES Y EQUIPOS UTILIZADOS PARA la Aplicación de Ultrasonido.

\begin{tabular}{|c|c|c|}
\hline PRODUCTO & $\begin{array}{l}\text { INTENSIDAD/ } \\
\text { TIEMPO }\end{array}$ & EQUIPO \\
\hline Leche & $\begin{array}{l}\text { Frecuencia: } 24 \mathrm{KHz} \text {, } \\
\text { - Amplitudes: } 0,40 \text {, } \\
72 \text { y } 120 \mu \mathrm{m}, \\
\text { - Temperatura: } 20- \\
57^{\circ} \mathrm{C} \text { - - Tiempo: } 30 \\
\text { minutos. }\end{array}$ & $\begin{array}{l}\text { Sonda } \\
\text { ultrasónica }\end{array}$ \\
\hline frutas & $\begin{array}{l}\text { Frecuencia: } \\
\text { 20KHz, } \\
\text { - Amplitud: } 24.4 \text { y } \\
\text { 61.0um, } \\
\text { - Tiempo: } 2 \text { a } 10 \\
\text { minutos. }\end{array}$ & $\begin{array}{l}\text { Túnel de } \\
\text { ultrasonido }\end{array}$ \\
\hline Zumos y leche & $\begin{array}{l}\text { Frecuencia: } 20 \mathrm{KHz}, \\
\text { - Potencia: } 100 \mathrm{~W}, \\
\text { - Temperatura: } 20 \mathrm{a} \\
57^{\circ} \mathrm{C} \text {. }\end{array}$ & $\begin{array}{l}\text { Sonda } \\
\text { ultrasónica }\end{array}$ \\
\hline $\begin{array}{l}\text { Aceites/In- } \\
\text { activación de } \\
\text { microorganis- } \\
\text { mos }\end{array}$ & $\begin{array}{l}\text { Frecuencia: } 20 \mathrm{KHz} \text {, } \\
\text { - Amplitudes: } 59.5 \text {, } \\
\text { - Tiempo: 2, 4, 6, } 8 \text { y } \\
10 \text { minutos. }\end{array}$ & $\begin{array}{l}\text { Túnel de } \\
\text { ultrasonido }\end{array}$ \\
\hline $\begin{array}{l}\text { Carnes pro- } \\
\text { cesadas y de } \\
\text { pieza entera }\end{array}$ & $\begin{array}{l}\text { Frecuencias: } 20 \mathrm{a} \\
35 \mathrm{KHz} \text {. }\end{array}$ & $\begin{array}{l}\text { Sonda } \\
\text { ultrasónica }\end{array}$ \\
\hline Secado & $\begin{array}{l}\text { Frecuencia: } 20 \mathrm{KHz} \text {, } \\
\text { Tiempo: } 60 \mathrm{~min}\end{array}$ & $\begin{array}{l}\text { Secador con } \\
\text { bandeja }\end{array}$ \\
\hline
\end{tabular}

Fuente El autor

\section{Conclusiones}

- La aplicación del ultrasonido en la industria de los alimentos es una tecnología reciente, con buenos resultados para la inactivación de microorganismos, mejoramiento de los procesos térmicos y conservación de las características nutricionales.

- Las principales aplicaciones del ultrasonido en la industria de alimentos son: inactivación enzimática, filtración, emulsificación, desgasificación de líquidos y homogenización.

- Los efectos que producen los ultrasonidos de potencia sobre los medios en que se aplican pueden influir en los procesos de transferencia de materia en sistemas sólido/líquido y sólido/ gas. La influencia puede darse tanto en la parte del fluido (resistencia externa) como en la parte del sólido (resistencia interna).

- La frecuencia más aplicada en la industria de los alimentos, está en el rango de 18 y $100 \mathrm{kHz}$ de potencia, siendo $24 \mathrm{KHz}$ la más efectiva para la inactivación enzimática y celular.

- Frecuencias mayores a $100 \mathrm{kHz}$ de intensidad acústica producida por el ultrasonido no es recomendable para la inactivacion de microorganismos.

\section{REFERENCIAS}

[1] G. V. Bermúdez-Aguirre, D. yBarbosa-Canovas, "Study of butter fat content in milk on the inactivation of Listeria innocua ATCC 51742 by thermo-sonication," Innovative Food Science and Emerging Technologies, vol. 9, pp. 176-185, 2008.

[2] S. Arroyo,C., Cebrián, G., Pagán, R. y Condón, "Inactivation of Cronobacter sakazakii by ultrasonic waves under pressure in buffer and foods," International Journal of Food Microbiology, , vol. 144, pp. 446-454, 2011.

[3] A. R. Jambrak, T. J. Mason, V. Lelas, Z. Herceg, and I. L. Herceg, "Effect of ultrasound treatment on solubility and foaming properties of whey protein suspensions," Journal of Food Engineering, vol. 86, no. 2, pp. 281-287, May 2008.

[4] G. L. Brown, T., James, S.J. y Purnell, "Cutting forces in foods: experimental measurements," Journal of Food Engineering, vol. 70, pp. 165-170, 2005.

[5] T.Bosiljkov, "Impact ultrasound-enhanced homogenizacion on physicla properties of soybean milk," vol. 17, pp. 1029-1034, 2009.

[6] T. Demirdôven, A. y Baysal, "The Use of Ultrasound and CombinedTechnologies in Food Preservation," Food Reviews International, vol. 25, pp. 1-11, 2009.

[7] [J. N. Coupland, "Low intensity ultrasound," Food Research International, vol. 37, pp. 537-543, 2004.

[8] E. T. Rodgers, S. L. \& Ryser, "Reduction of microbial pathogens during apple cider production using sodium hypochlorite, copper ion, and sonication," and sonication. J Food Prot, vol. 67(4), pp. 766-771, 2004.

[9] S. Ugarte-Romero, E., Feng, H., Martin, S.E., Cadwallader, K.R., Robinson, "Inactivation of Escherichia coli with power ultrasound in apple cider," J Food Sci, vol. 71(2), pp. 102-108, 2006.

[10] AM Herrero; MD Romero de Avila, "Innovaciones en el procesado de alimentos: Tecnologías no térmicas," vol. 50, pp. 71-74, 2006. 
[11] R. M. Earnshaw, R. G., Appleyard, J. y Hurst, "Understanding physical inactivation processes: combined preservation opportunities using heat, ultrasound and pressure," International Journal of Food Microbiology, vol. 28, pp. 197-219, 1995.

[12] C. Ashokkumar, M., Sunartio, D., Kentish, S., Mawson, R., Simons, L., Vilkhu, K. y Versteeg, "Modification of food ingredients by ultrasound to improve funcionality: A preliminary study on a model system. Innovative," Innovative Food Science and Emerging Technologics, vol. 9, pp. 155-160, 2008.

[13] T. Cameron, M., McMaster y L., Britz, "Electron microscopic analysis of dairy microbes inactivated by ultrasound," Ultrasonics Sonochemistry, vol. 15, pp. 960-964, 2008.

[14] J. Nathaly, P. Montero, C. Andrés, R. Jiménez, B. Daniel, Y. Villarreal, P. D. I. De Alimentos, D. De Ingeniería, U. Jorge, and T. Lozano, "Castilla ( Rubus glaucus Benth ): Efecto sobre la calidad funcional y evaluación como pretratamiento al secado convectivo," pp. 15-38.

[15] R. Brnčić, "Textural properties of infra red dried apple slices as affected by high power ultrasound pretreatment," vol. 9, no. 41, pp. 6907-6915, 2010.

[16] M. Chemat, F., Huma, Z y Khan, "Applications of ultrasound in food technology: Processing, preservation and extraction," Ultrasonics Sonochemistry, vol. 18, pp. 813-835, 2011.

[17] G. Gonz, "Aplicaciónes del ultrasonido para la estabilización de sistemas alimentarios," 2011.

[18] [18]G. F. Barbero, a Liazid, M. Palma, and C. G. Barroso, "Ultrasound-assisted extraction of capsaicinoids from peppers.", Talanta, vol. 75, no. 5, pp. 1332-7, Jun. 2008.

[19] E. Gallego-Juarez, J.A., Rodrigues-Corral, G., Acosta- Aparicio, V.M., Andres-Gallego and F. BlancoBlanco, A. y Montoya-Vitini, "Procedimiento y sistema ultrasónico de desespumación mediante emisores con placa vibrante escalonada," U.S. Patent 2002 021132012.

[20] T. Lida, Y., Tuziuti, T., Yasui, K., Towata, A. y Kosuka, "Control of viscosity in starch and polysaccharide solutions with ultrasound after gelatinization," Innovative Food Science and Emerging Technologies, vol. 9, pp. 140-146, 2008.

[21] B. K. Tiwari, K. Muthukumarappan, C. P. O’Donnell, and P. J. Cullen, "Inactivation kinetics of pectin methylesterase and cloud retention in sonicated orange juice," Innovative Food Science \& Emerging Technologies, vol. 10, no. 2, pp. 166-171, Apr. 2009.

[22] M. Yaldagard, S. A. Mortazavi, and F. Tabatabaie, "Influence of ultrasonic stimulation on the germination of barley seed and its alpha-amylase activity," vol. 7, no. 14, pp. 2465-2471, 2008.

[23] K. Vilkhu, R. Mawson, L. Simons, and D. Bates, "Applications and opportunities for ultrasound assisted extraction in the food industry - A review," 2001.
[24] H. Arnold, G., Leiteritz, S., Zahn, S. y Rohm, "Ultrasonic cutting of cheese: composition affects cutting work reduction and energy demand," International Dairy Journal, vol. 19, pp. 314-320, 2009.

[25] J. R. WU, H. Hulbert, G.J. y Mount, "Effects of ultrasound on milk homogenization and fermentation with yogurt starter," Innovative Food Science and Emerging Technologies, vol. 1, pp. 211-218, 2000.

[26] P. Mongenot, N., Charrier, S. y Chalier, "Effect of ultrasound emulsification on cheese aroma encapsulation by carbohydrates," Journal of Agricultural Food Chemistry, vol. 48, pp. 861- 867, 2000.

[27] S. Martini, a. H. Suzuki, and R. W. Hartel, "Effect of High Intensity Ultrasound on Crystallization Behavior of Anhydrous Milk Fat," Journal of the American Oil Chemists' Society, vol. 85, no. 7, pp. 621-628, May 2008.

[28] B. y S. D. W. Li, "Novel methods for rapid freezing and thawing of foods-a review," Journal of Food Engineering, vol. 54, pp. 175-182, 2009.

[29] H. LI, L. Pordesimo, and J. Weiss, "High intensity ultrasound-assisted extraction of oil from soybeans," Food Research International, vol. 37, no. 7, pp. 731738, Aug. 2004.

[30] H. Floros, J. D., y Liang, "Acoustically assisted diffusion through membranes and biomaterials: High-intensity ultrasound accelerates diffusion and can be used to improve food processes," Food Technology, p. 79, 1994.

[31] T. C. S. Cohen, J.S. y Yang, "Progress in food dehydration," Trend food Science Technology, vol. 6, pp. 20-25, 1995.

[32] Zbigniew J;, J. Stadnik, and D. Stasiak, "APPLICATIONS OF ULTRASOUND IN FOOD TECHNOLOGY Zbigniew J. Dolatowski, Joanna Stadnik, Dariusz Stasiak," 2007.

[33] P. J. O’Donnell, C.P. Tiwari, B.K., Bourke, P. y Cullen, "Effect of ultrasonic processing on food enzymes of industrial importance," Trends in Food Science y Technology, vol. 21, pp. 358-367, 2010.

[34] D. J. Pejin, L. V. Mojović, J. D. Pejin, O. S. Grujić, S. L. Markov, S. B. Nikolić, and M. N. Marković, "Increase in bioethanol production yield from triticale by simultaneous saccharification and fermentation with application of ultrasound," Journal of Chemical Technology \& Biotechnology, vol. 87, no. 2, pp. 170-176, Feb. 2012.

[35] S. Patil, P. Bourke, B. Cullen, J. M. Frias, and P. J. Cullen, "The Effects of Acid Adaptation on Escherichia Coli Inactivation Using Power Ultrasound," pp. 0-26, 2009.

[36] C. BEndicho, I. De La Calle, F. Pena, M. Costas, N. Cabaleiro, and I. Lavilla, "Ultrasound-assisted pretreatment of solid samples in the context of green analytical chemistry," TrAC Trends in Analytical Chemistry, vol. 31, pp. 50-60, Jan. 2012. 
[37] J. Wu, T. V. Gamage, K. S. Vilkhu, L. K. Simons, and R. Mawson, "Effect of thermosonication on quality improvement of tomato juice," Innovative Food Science \& Emerging Technologies, vol. 9, no. 2, pp. 186-195, Apr. 2008.

[38] M. Zuo, J.Y., Knoerzer, K., Mawson, R., Kentish, S. y Ashokkumar, "The pasting properties of sonicated waxy rice starch suspensions," Ultrasonic sonochemistry, vol. 16, pp. 462-468, 2009.

[39] M. Yaldagard, S. A. Mortazavi, F. Tabatabaie, and J. I. Brew, "Application of Ultrasonic Waves as a Priming Technique for Accelerating and Enhancing the Germination of Barley Seed : Optimization of Method by the Taguchi Approach," pp. 14-21.

[40] J. Garcia-Noguera, F. I. P. Oliveira, M. I. Gallão, C. L. Weller, S. Rodrigues, and F. a. N. Fernandes, "Ultrasound-Assisted Osmotic Dehydration of Strawberries: Effect of Pretreatment Time and Ultrasonic Frequency," Drying Technology, vol. 28, no. 2, pp. 294-303, Mar. 2010.

[41] [41]B. Zhou, H. Feng, and Y. Luo, "Ultrasound enhanced sanitizer efficacy in reduction of Escherichia coli O157:H7 population on spinach leaves.," Journal of food science, vol. 74, no. 6, pp. M308-13, Aug. 2009.
[42] [42] Roberts R.T., "High intensity ultrasonics in food processing," Chemical Industry, vol. 15, pp. 119$121,1993$.

[43] [43]P. J. Tiwari, B.K., Muthukumarappana, K., O'Donella, C.P. y Cullen, "Innovative Food Science and Emerging Technologies," vol. 10, pp. 166-171, 2009.

[44] [44]A. Mortazavi and F. Tabatabaie, "Study of Ice Cream Freezing Process after Treatment with Ultrasound," World Applied Sciences Journal, vol. 4, no. 2, pp. 188-190, 2008.

[45] [45]K. S. Suslick, "In: Ultrasound:Chemical, Biological and Physical Effects," Biological and Physical Effects, pp. 123-163, 1998.

[46] [46] L. Zheng and D. Sun, "Innovative appli- cations of power ultrasound during food freezing processes $-a$ review," 1999.

[47] [47] H. Feng, W. Yang, and T. Hielscher, "Power Ultrasound," Food Science and Technology International, vol. 14, no. 5, pp. 433-436, Oct. 2008.

[48] [48]G. Hunter, M. Lucas, I. Watson, and R. Parton, "A radial mode ultrasonic horn for the inactivation of Escherichia coli K12.," Ultrasonics sonochemistry, vol. 15, no. 2, pp. 101-9, Feb. 2008. 